H. Morikawa

Nagoya Math. J.

Vol. 96 (1984), 113-126

\title{
A DECOMPOSITION THEOREM ON DIFFERENTIAL POLYNOMIALS OF THETA FUNCTIONS
}

\author{
HISASI MORIKAWA
}

Let $\tau=\left(\tau_{i j}\right)$ be a symmetric complex $g \times g$ matrix with the positive definite imaginary part. A theta function of level $n$ means an entire function $f(z)$ in $g$ complex variables $z=\left(z_{1}, \cdots, z_{g}\right)$ satisfying the difference relations:

$$
f(z+\hat{b}+b \tau)=\exp \left(-\pi n \sqrt{-1}\left(b \tau^{t} b+2 z^{t} b\right)\right) f(z), \quad\left((\hat{b}, b) \in Z^{g} \times Z^{g}\right) .
$$

Denoting by $\Theta_{0}^{(n)}$ the vector space of theta functions of level $n$, we get the graded algebra of theta functions;

$$
\Theta_{0}=\sum_{n \geq 1} \Theta_{0}^{(n)}
$$

Theta series

$$
\begin{gathered}
\vartheta^{(n)}\left[\begin{array}{c}
a / n \\
0
\end{array}\right](\tau \mid z)=\sum_{\ell \in \boldsymbol{Z}^{g}} \exp \left(\pi n \sqrt{-1}\left(\left(\ell+\frac{a}{n}\right) \tau^{t}\left(\ell+\frac{a}{n}\right)+2 z^{t}\left(\ell+\frac{a}{n}\right)\right)\right), \\
\left(a \in \boldsymbol{Z}^{g} / n \boldsymbol{Z}^{g}\right)
\end{gathered}
$$

form a canonical basis of $\Theta_{0}^{(n)}$, and thus

$$
\operatorname{dim} \Theta_{0}^{(n)}=n^{g} .
$$

In the present article we shall prove the following decomposition theorem:

The algebra of differential polynomials of theta functions has a canonical linear basis

$$
\left\{\left(\frac{\partial}{\partial z}\right)^{j} \vartheta^{(n)}\left[\begin{array}{c}
a / n \\
0
\end{array}\right](\tau \mid z) \mid j \in Z_{\geq 0}^{g}, a \in Z^{g} / n Z, n \geq 1\right\},
$$

i.e. any differential polynomial is uniquely expressed as a linear combination of $(\partial / \partial z)^{j} \vartheta^{(n)}\left[\begin{array}{c}a / n \\ 0\end{array}\right](\tau \mid z),\left(j \in Z_{\geq 0}^{g}, a \in Z^{g} / n Z^{g}, n \geq 1\right)$ with constant

Received November 14, 1983. 
coefficients depending on $\tau$. More precisely we have the explicit expressions of the components of the decomposition.

The key is a very similar idea as making transvectants in the classical invariant theory, however the Lie algebra is Heisenberg Lie algebra instead of $s \ell_{2}$. The algebra $\Theta_{0}$ of theta functions is embedded in a graded algebra $\Theta$ of auxiliary theta functions in $2 g$ complex variables $(u, z)=$ $\left(u_{1}, \cdots, u_{g}, z_{1}, \cdots, z_{g}\right)$ with the following properties,

$1^{\circ} \quad$ A realization $\left\langle\mathscr{E}, \mathscr{D}_{1}, \cdots, \mathscr{D}_{g}, \Delta_{1}, \cdots, \Delta_{g}\right\rangle$ of Heisenberg Lie algebra acts on $\Theta$ as derivations,

$2^{\circ} \Theta_{0}$ is the subalgebra consisting of all the elements $\varphi$ such that $\mathscr{D}_{i} \varphi=0(1 \leq i \leq g)$,

$3^{\circ} \quad\left\{\Delta^{j} \vartheta^{(n)}\left[\begin{array}{c}a / n \\ 0\end{array}\right](\tau \mid z) \mid j \in Z_{\geq 0}^{g}, a \in Z^{g} / n Z^{g}, n \geq 1\right\}$ is a canonical linear basis of $\Theta$,

$4^{\circ}$ The mapping

$$
\Delta^{j} \vartheta^{(n)}\left[\begin{array}{c}
a / n \\
0
\end{array}\right](\tau \mid z) \longrightarrow\left(\frac{\partial}{\partial z}\right)^{j} \vartheta^{(n)}\left[\begin{array}{c}
a / n \\
0
\end{array}\right](\tau \mid z), \quad\left(j \in Z_{\geq 0}^{g}, a \in Z^{g} / n Z^{g}, n \geq 1\right)
$$

induces an algebra isomorphism of $\Theta$ onto the algebra of differential polynomials of theta functions.

We shall also characterize differential polynomials of theta functions which are theta functions.

The associative law for the structure constants of

$$
C\left[\cdots,\left(\frac{\partial}{\partial z}\right)^{j} \vartheta^{(n)}\left[\begin{array}{c}
a / n \\
0
\end{array}\right](\tau \mid z), \cdots\right]
$$

with respect to the basis must be very important relations between

$$
\left\{\left(\frac{\partial}{\partial z}\right)^{j} \vartheta^{(n)}\left[\begin{array}{c}
a / n \\
0
\end{array}\right]\left(\tau \mid \frac{\hat{a}}{n}\right) \mid j \in Z_{\geq 0}^{g} ; a, \hat{a} \in Z^{g} / n Z^{g} ; n \geq 1\right\} .
$$

\section{Notations.}

$Z_{\geq 0}=\{$ non-negative integers $\}, Z_{\geq 0}^{g}=\left\{j=\left(j_{1}, \cdots, j_{g}\right) \mid j_{i} \in Z_{\geqslant 0}\right\}$, $j \pm \varepsilon_{i}=\left(j_{1}, \cdots, j_{i-1}, j_{i} \pm 1, j_{i+1}, \cdots, j_{g}\right), j !=j_{1} ! \cdots j_{g} !$, $\left(\begin{array}{l}j \\ p\end{array}\right)=\left(\begin{array}{c}j_{1} \\ p_{1}\end{array}\right) \cdots\left(\begin{array}{c}j_{g} \\ p_{g}\end{array}\right),\left(\begin{array}{c}j \\ k^{(1)}, \cdots, k^{(r)}\end{array}\right)=\left(\begin{array}{c}j_{1} \\ k_{1}^{(1)}, \cdots, k_{1}^{(r)}\end{array}\right) \cdots\left(\begin{array}{c}j_{g} \\ k_{g}^{(1)}, \cdots, k_{g}^{(r)}\end{array}\right)$, $|j|=j_{1}+\cdots+j_{g}, u=\left(u_{1}, \cdots, u_{g}\right), z=\left(z_{1}, \cdots, z_{g}\right), u^{j}=u_{1}^{j_{1}} \cdots u_{g}^{j^{g}}$, $\boldsymbol{z}^{j}=\boldsymbol{z}_{1}^{j_{1}}, \cdots, \boldsymbol{z}_{g}^{j_{g}}$, $\left(\frac{\partial}{\partial u}\right)^{j}=\left(\frac{\partial}{\partial u_{1}}\right)^{j_{1}} \cdots\left(\frac{\partial}{\partial u_{g}}\right)^{j_{g}},\left(\frac{\partial}{\partial z}\right)^{j}=\left(\frac{\partial}{\partial z_{1}}\right)^{j_{1}} \cdots\left(\frac{\partial}{\partial z_{g}}\right)^{j_{g}}$, 


$$
\left(2 \pi n \sqrt{-1} u+\frac{\partial}{\partial u}\right)^{j}=\left(2 \pi n \sqrt{-1} u_{1}+\frac{\partial}{\partial z_{1}}\right)^{j_{1}} \cdots\left(2 \pi n \sqrt{-1} u_{g}+\frac{\partial}{\partial z_{g}}\right)^{j_{g}} .
$$

\section{§1. Auxiliary theta functions}

1.1. An auxiliary theta function of level $n$ means a function $\varphi(u, z)$ in $2 g$ complex variables $(u, z)=\left(u_{1}, \cdots, u_{g}, z_{1}, \cdots, z_{g}\right)$ such that

$1^{\circ} \varphi(u, z)$ is a polynomial in $u=\left(u_{1}, \cdots, u_{g}\right)$ whose coefficients are entire functions in $z=\left(z_{1}, \cdots, z_{g}\right)$,

$$
2^{\circ} \varphi(u+b, z+\hat{b}+b \tau)=\exp \left(-\pi n \sqrt{-1}\left(b \tau^{t} b+2 z^{t} b\right)\right) \varphi(u, z),((\hat{b}, b) \epsilon
$$
$\left.\boldsymbol{Z}^{g} \times \boldsymbol{Z}^{g}\right)$.

Denoting by $\Theta^{(n)}$ the vector space of auxiliary theta functions of level $n$, we obtain a graded algebra

$$
\Theta=\sum_{n \geq 1} \Theta^{(n)}
$$

of auxiliary theta functions, which contains the graded algebra $\Theta_{0}$ of theta functions as the subalgebra of polynomials of degree zero in $u$. Auxiliary theta series are also defined as follows,

$$
\begin{aligned}
& \vartheta_{j}^{(n)}\left[\begin{array}{c}
a / n \\
0
\end{array}\right](\tau \mid u, z) \\
& =(2 \pi n \sqrt{-1})^{|j|} \sum_{\ell \in Z^{g}}\left(u+\ell+\frac{a}{n}\right)^{j} \\
& \quad \cdot \exp \pi n \sqrt{-1}\left(\left(\ell+\frac{a}{n}\right) \tau^{t}\left(\ell+\frac{a}{n}\right)+2 z^{t}\left(\ell+\frac{a}{n}\right)\right) \\
& \quad\left(j \in Z_{\geq 0}^{g}, a \in Z^{g} / n Z^{g}, n \geq 1\right) .
\end{aligned}
$$

Lemma 1.1.

$$
\begin{gathered}
\vartheta_{j}^{(n)}\left[\begin{array}{c}
a / n \\
0
\end{array}\right](\tau \mid u, z)=\left(2 \pi n \sqrt{-1} u+\frac{\partial}{\partial z}\right)^{j} \vartheta^{(n)}\left[\begin{array}{c}
a / n \\
0
\end{array}\right](\tau \mid z) \\
\vartheta_{j}^{(n)}\left[\begin{array}{c}
a / n \\
0
\end{array}\right](\tau \mid u+b, z+\hat{b}+b \tau) \\
=\exp \left(-\pi n \sqrt{-1}\left(b \tau^{t} b+2 z^{t} b\right)\right) \vartheta_{j}^{(n)}\left[\begin{array}{c}
a / n \\
0
\end{array}\right](\tau \mid u, z) \\
\left.(\hat{b}, b) \in Z^{g} \times Z^{g}\right) .
\end{gathered}
$$

Proof. For $a, b, \hat{b}$ in $Z^{g}$ we have 


$$
\begin{aligned}
& \left(2 \pi n \sqrt{-1} u+\frac{\partial}{\partial z}\right)^{j} \exp \left(\pi n \sqrt{-1}\left(\left(\ell+\frac{a}{n}\right) \tau^{t}\left(\ell+\frac{a}{n}\right)+2 z^{t}\left(\ell+\frac{a}{n}\right)\right)\right) \\
& =(2 \pi n \sqrt{-1})^{|j|}\left(u+\ell+\frac{a}{n}\right)^{j} \\
& \quad \exp \left(\pi n \sqrt{-1}\left(\left(\ell+\frac{a}{n}\right) \tau^{t}\left(\ell+\frac{a}{n}\right)+2 z\left(\ell+\frac{a}{n}\right)\right)\right) \\
& \left(u+\ell+b+\frac{a}{n}\right)^{j} \\
& \quad \cdot \exp \left(\pi n \sqrt{-1}\left(\left(\ell+b+\frac{a}{n}\right) \tau^{t}\left(\ell+b+\frac{a}{b}\right)+2 z^{t}\left(\ell+b+\frac{a}{n}\right)\right)\right) \\
& =\exp \left(\pi n \sqrt{-1}\left(b \tau^{t} b+2 z^{t} b\right)\left(u+\ell+b+\frac{a}{n}\right)^{j}\right. \\
& \quad \exp \left(\pi n \sqrt { - 1 } \left(\left(\ell+\frac{a}{n}\right) \tau^{t}\left(\ell+\frac{a}{n}\right) \tau^{t}\left(\ell+\frac{a}{n}\right)\right.\right. \\
& \left.\left.\quad+2(z+\hat{b}+b \tau)\left(\ell+\frac{a}{n}\right)\right)\right) .
\end{aligned}
$$

Hence, making the sum with respect to $\ell \in Z^{g}$, we obtain (1.2), (1.3).

THEOREM 1.1. $\left\{\vartheta_{j}^{(n)}\left[\begin{array}{c}a / n \\ 0\end{array}\right](\tau \mid u, z) \mid j \in Z_{20}^{g}, a \in Z^{g} / n Z^{g}\right\}$ is a basis of the space $\Theta^{n}$ of auxiliary theta functions of level $n$.

Proof. By virtue of Lemma $1.1 \vartheta_{j}^{(n)}\left[\begin{array}{c}a / n \\ 0\end{array}\right](\tau \mid u, z)\left(j \in Z_{20}^{g}, a \in Z^{g} / n Z^{g}\right)$ belong to $\Theta^{(n)}$, and obviously they are linearly independent. Let $\varphi(u, z)$ $=\sum_{j} u^{j} f_{j}(z)$ be an element of $\Theta^{(n)}$, and let $u^{k} f_{k}(z)$ be one of terms with maximal degree $k$ in $u$. Then, comparing the coefficients of $u^{k}$ in the both sides of

$$
\sum_{j}(u+b)^{j} f_{j}(z+\hat{b}+b \tau)=\exp \left(-\pi n \sqrt{-1}\left(b \tau^{t} b+2 z^{t} b\right)\right) \sum_{j} u^{j} f_{j}(z)
$$

we have

$$
f_{k}(z+\hat{b}+b \tau)=\exp \left(-\pi n \sqrt{-1}\left(b \tau^{t} b+2 z^{t} b\right)\right) f_{k}(z) .
$$

This means that there exists a system $\left(\alpha_{a}\right)_{a \in Z^{g} / n Z^{g}}$ of constants such that

$$
f_{k}(z)=\sum_{a} \alpha_{a} \vartheta^{(n)}\left[\begin{array}{c}
a / n \\
0
\end{array}\right](\tau \mid z),
$$

and thus 


$$
\varphi(u, z)-\sum_{a} \alpha_{a} \vartheta_{k}^{(n)}\left[\begin{array}{c}
a / n \\
0
\end{array}\right](\tau \mid u, z)
$$

is an element in $\Theta^{(n)}$ without $u^{k}$-term and all the new terms are of lower degree than $k$ in $u$. Proceeding this process successively, we can express $\varphi(u, z)$ as a linear sum of $\vartheta_{j}^{(n)}\left[\begin{array}{c}a / n \\ 0\end{array}\right](\tau \mid u, z)\left(j \in Z_{\geq 0}^{g}, a \in Z^{g} / n Z^{g}\right)$.

1.2. Denoting the projection operators by

$$
\sigma^{(n)}: \Theta \longrightarrow \Theta^{(n)}, \quad(n \geq 1)
$$

we define differential operators

$$
\begin{aligned}
& \mathscr{E}=\sum_{n \geq 1} n \sigma^{(n)}, \\
& \mathscr{D}_{i}=\sum_{n \geq 1} \frac{1}{2 \pi \sqrt{-1}} \frac{\partial}{\partial u_{i}} \circ \sigma^{(n)}, \\
& \Delta_{i}=\sum_{n \geq 1}\left(2 \pi n \sqrt{-1} u_{i}+\frac{\partial}{\partial z_{i}}\right) \circ \sigma^{(n)}, \\
& \mathscr{D}^{j}=\mathscr{D}_{1}^{j_{1}} \ldots \mathscr{D}_{g}^{j_{g} g}, \quad \Delta_{1}^{j_{1}} \cdots \Delta_{g}^{j g} .
\end{aligned}
$$

Proposition 1.1.

$$
\begin{gathered}
\mathscr{D}_{i} \vartheta_{j}^{(n)}\left[\begin{array}{c}
a / n \\
0
\end{array}\right](\tau \mid u, z)=n j_{i} \vartheta_{j-\varepsilon_{i}}^{(n)}\left[\begin{array}{c}
a / n \\
0
\end{array}\right](\tau \mid u, z), \\
\Delta_{i} \vartheta_{j}^{(n)}\left[\begin{array}{c}
a / n \\
0
\end{array}\right](\tau \mid u, z)=\vartheta_{j+\varepsilon_{i}}^{(n)}\left[\begin{array}{c}
a / n \\
0
\end{array}\right](\tau \mid u, z), \\
\vartheta_{j}^{(n)}\left[\begin{array}{c}
a / n \\
0
\end{array}\right](\tau \mid u, z)=\Delta^{j} \vartheta^{(n)}\left[\begin{array}{c}
a / n \\
0
\end{array}\right](\tau \mid z), \\
\frac{1}{p !} \mathscr{D}^{p} \vartheta_{j}^{(n)}\left[\begin{array}{c}
a / n \\
0
\end{array}\right](\tau \mid u, z)=\left(\begin{array}{c}
j \\
p
\end{array}\right) n^{|p|} \vartheta_{j-p}^{(n)}\left[\begin{array}{c}
a / n \\
0
\end{array}\right](\tau \mid u, z), \\
\frac{1}{j !} \mathscr{D}^{j} \vartheta_{j}^{(n)}\left[\begin{array}{c}
a / n \\
0
\end{array}\right](\tau \mid u, z)=n^{|j|} \vartheta^{(n)}\left[\begin{array}{c}
a / n \\
0
\end{array}\right](\tau \mid z) \\
\left(j, p \in Z_{\geq 0}^{g}, j \geq p, a \in Z^{g} / n Z^{g}, n \geq 1\right) .
\end{gathered}
$$

Proof. From the expression

$$
\vartheta_{j}^{(n)}\left[\begin{array}{c}
a / n \\
0
\end{array}\right](\tau \mid u, z)=\left(2 \pi n \sqrt{-1} u+\frac{\partial}{\partial z}\right)^{j} \vartheta^{(n)}\left[\begin{array}{c}
a / n \\
0
\end{array}\right](\tau \mid z)
$$

it follows (1.4), (1.5), (1.6). Applying (1.4) and (1.5) successively, we have (1.7), (1.8). 
Proposition 1.2. $\mathscr{E}, \mathscr{D}_{1}, \cdots, \mathscr{D}_{g}, \Delta_{1}, \cdots, \Delta_{g}$ are derivations of $\Theta$ such that

$$
\begin{aligned}
& {\left[\mathscr{E}, \mathscr{D}_{i}\right]=\left[\mathscr{E}, \Delta_{i}\right]=\left[\mathscr{D}_{i}, \mathscr{D}_{j}\right]=\left[\Delta_{i}, \Delta_{j}\right]=0,} \\
& {\left[\mathscr{D}_{i}, \Delta_{i^{\prime}}\right]=\left\{\begin{array}{ll}
\mathscr{E} & \left(i=i^{\prime}\right) \\
0 & \left(i \neq i^{\prime}\right)
\end{array} \quad\left(1 \leq i, i^{\prime}, j \leq g\right) .\right.}
\end{aligned}
$$

Proof. By virtue of Proposition $1.2 \mathscr{E}, \mathscr{D}_{1}, \cdots, \mathscr{D}_{g}, \Delta_{1}, \cdots, \Delta_{g}$, map $\Theta$ into itself. Since $\Theta=\sum_{n \geq 1} \Theta^{(n)}$ is a graded algebra, $\mathscr{E}, \mathscr{D}_{1}, \cdots, \mathscr{D}_{g}$, $\Delta_{1}, \cdots, \Delta_{g}$ are derivations of $\Theta$. By simple calculation we have (1.9).

Proposition 1.2 states $\left\langle\mathscr{E}, \mathscr{D}_{1}, \cdots, \mathscr{D}_{g}, \Delta_{1}, \cdots, \Delta_{g}\right\rangle$ is a realization of Heisenberg Lie algebra acting on $\Theta$ as derivations.

Proposition 1.3. The graded algebra of theta functions is the subalgebra consisting of all the elements $\varphi$ such that $\mathscr{D}_{i} \varphi=0(1 \leq i \leq g)$.

Proof. Each $\phi$ in $\Theta_{0}$ contains no $u_{i}$ and

$$
\mathscr{D}_{i}=\sum_{n \geq 1} \frac{1}{2 \pi \sqrt{-1}} \frac{\partial}{\partial u_{i}} \circ \sigma^{(n)} \quad(1 \leq i \leq g),
$$

hence we have $\mathscr{D}_{i} \varphi=0(1 \leq i \leq g)$. Conversely, assume

$$
\mathscr{D}_{i}\left(\sum \alpha_{j, a / n, n} \vartheta_{j}^{(n)}\left[\begin{array}{c}
a / n \\
0
\end{array}\right](\tau \mid u, z)\right)=0 \quad(1 \leq i \leq g) .
$$

Then it follows

$$
\sum n j_{i} \alpha_{j, a / n, n} \vartheta_{j-\varepsilon_{i}}^{(n)}\left[\begin{array}{c}
a / n \\
0
\end{array}\right](\tau \mid u, z)=0 \quad(1 \leq i \leq g) .
$$

This means $\alpha_{j, a / n, n}=0$ for $j \neq 0$.

\section{§2. Projection operators}

2.1. In order to express the projection operators

$$
\sigma_{j}^{(n)}: \Theta \longrightarrow \Delta^{j} \Theta_{0}^{(n)} \quad\left(j \in Z_{\geq 0}^{g}, n \geq 1\right),
$$

we need a lemma.

LEMMA 2.1.

$$
\left(\sum_{p \leq k} \frac{(-1)^{|p|}}{p !} n^{-|p|} \Delta^{p} \mathscr{D}^{p}\right) \vartheta_{k}^{(n)}\left[\begin{array}{c}
a / n \\
0
\end{array}\right](\tau \mid u, z)=\left\{\begin{array}{cc}
\vartheta^{(n)}\left[\begin{array}{c}
a / n \\
0
\end{array}\right](\tau \mid z) & (k=0) \\
0 & (k \neq 0)
\end{array}\right.
$$




$$
\begin{gathered}
\left(\Delta^{j}\left(\sum_{p} \frac{(-1)^{|p|}}{p !} n^{-|p|} \Delta^{p} \mathscr{D}^{p}\right) \frac{1}{j !} n^{-|j|} \mathscr{D}^{j}\right) \vartheta_{k}^{(n)}\left[\begin{array}{c}
a / n \\
0
\end{array}\right](\tau \mid u, z) \\
=\left\{\begin{array}{cc}
\vartheta_{j}^{(n)}\left[\begin{array}{c}
a / n \\
0
\end{array}\right](\tau \mid u, z) & (k=j) \\
0 & (k \neq j)
\end{array}\right. \\
\left(j, k \in Z_{\geq 0}^{g}, \quad a \in Z^{g} / n Z^{g}, n \geq 1\right) .
\end{gathered}
$$

Proof. From (1.4), (1.5), (1.6), (1.7) it follows

$$
\begin{aligned}
& \left(\sum_{p} \frac{(-1)^{|p|}}{p !} n^{-|p|} \Delta^{p} \mathscr{D}^{p}\right) \vartheta_{k}^{(n)}\left[\begin{array}{c}
a / n \\
0
\end{array}\right](\tau \mid u, z) \\
& =\sum_{p \leq k}(-1)^{|p|}\left(\begin{array}{l}
k \\
p
\end{array}\right) \Delta^{p} \vartheta_{k-p}^{(n)}\left[\begin{array}{c}
a / n \\
0
\end{array}\right](\tau \mid u, z) \\
& =\left(\sum_{p \leq k_{,}}(-1)^{|p|}\left(\begin{array}{l}
k \\
p
\end{array}\right)\right) \cdot \vartheta_{k}^{(n)}\left[\begin{array}{c}
a / n \\
0
\end{array}\right](\tau \mid u, z) \\
& =\left\{\begin{array}{cc}
\vartheta^{(n)}\left[\begin{array}{c}
a / n \\
0
\end{array}\right](\tau \mid z) & (k=0) \\
0 & (k \neq 0)
\end{array},\right. \\
& \left(\Delta^{j}\left(\sum_{p} \frac{(-1)^{|p|}}{p !} n^{-|p|} \Delta^{p} \mathscr{D}^{p}\right) \frac{1}{j !} n^{-|j|} \mathscr{D}^{j}\right) \vartheta_{k}^{(n)}\left[\begin{array}{c}
a / n \\
0
\end{array}\right](\tau \mid u, z) \\
& =\Delta^{j}\left(\sum_{p} \frac{(-1)^{|p|}}{p !} n^{-|p|} \Delta^{p} \mathscr{D}^{p}\right)\left(\begin{array}{c}
k \\
j
\end{array}\right) \vartheta_{k-j}^{(n)}\left[\begin{array}{c}
a / n \\
0
\end{array}\right](\tau \mid u, z) \\
& =\left(\begin{array}{c}
k \\
j
\end{array}\right) \Delta^{j}\left(\sum_{p} \frac{(-1)^{|p|}}{p !} n^{-|p|} \Delta^{p} \mathscr{D}^{p}\right) \vartheta_{k-j}^{(n)}\left[\begin{array}{c}
a / n \\
0
\end{array}\right](\tau \mid u, z) \\
& =\left\{\begin{array}{cc}
\Delta^{j} \vartheta^{(n)}\left[\begin{array}{c}
a / n \\
0
\end{array}\right](\tau \mid z)=\vartheta_{j}^{(n)}\left[\begin{array}{c}
a / n \\
0
\end{array}\right](\tau \mid u, z) & (j=k) \\
0 & (j \neq k)
\end{array}\right.
\end{aligned}
$$

Theorem 2.1. $\Theta$ has the direct sum decomposition

$$
\Theta=\sum_{i \in \mathbb{Z}_{\geq 0}^{g}} \Delta^{j} \Theta_{0}=\sum_{n \geq 1} \sum_{j \in Z_{\geq 0}^{g}} \Delta^{j} \Theta_{0}^{(n)}
$$

such that $\Delta^{j}$ induces a vector space isomorphism of $\Theta_{0}^{(n)}$ onto $\Delta^{j} \Theta_{0}^{(n)}$. The projection operators

$$
\sigma_{j}^{(n)}: \Theta \longrightarrow \Delta^{j} \Theta_{0}^{(n)}
$$

are given by

$$
\begin{gathered}
\sigma_{j}^{(n)}=\Delta^{j}\left(\sum_{p} \frac{(-1)^{|p|}}{p !} n^{-|p|} \Delta^{p} \mathscr{D}^{p}\right) \frac{1}{j !} n^{-|j|} \mathscr{D}^{j} \circ \sigma^{(n)} \\
\left(j \in Z_{\geq 0}^{g}, n \geq 1\right) .
\end{gathered}
$$


Proof. The first part of the assertion is a direct consequence of the fact: $\left\{\vartheta_{j}^{(n)}\left[\begin{array}{c}a / n \\ 0\end{array}\right](\tau \mid u, z) \mid j \in Z_{\geq 0}^{g}, \quad a \in Z^{g} / n Z^{g}, \quad n \geq 1\right\},\left\{\vartheta_{j}^{(n)}\left[\begin{array}{c}a / n \\ 0\end{array}\right](\tau \mid u, z) \mid a \in\right.$ $\left.Z^{g} / n Z^{g}\right\}$ and $\left\{\vartheta^{(n)}\left[\begin{array}{c}a / n \\ 0\end{array}\right](\tau \mid z) \mid a \in Z^{g} / n Z^{g}\right\}$ are the basis of $\Theta, \Delta^{j} \Theta_{0}^{(n)}$ and $\Theta_{0}^{(n)}$, respectively. The expression (2.4) is a direct consequence of (2.2).

CoRollary. The inverse mapping of $\Delta^{j}: \Theta_{0}^{(n)} \rightarrow \Delta^{j} \Theta_{0}^{(n)}$ is given by

$$
\left(\sum_{p} \frac{(-1)^{|p|}}{p !} n^{-|p|} \Delta^{p} \mathscr{D}^{p}\right) \frac{1}{j !} n^{-|j| \mathscr{D}^{j}} \quad\left(j \in Z_{\geq 0}^{g}, n \geq 1\right) .
$$

Proof. Since the mapping

$$
\vartheta^{(n)}\left[\begin{array}{c}
a / n \\
0
\end{array}\right](\tau \mid z) \longrightarrow \Delta^{j} \vartheta^{(n)}\left[\begin{array}{c}
a / n \\
0
\end{array}\right](\tau \mid z)=\vartheta_{j}^{(n)}\left[\begin{array}{c}
a / n \\
0
\end{array}\right](\tau \mid u, z)
$$

is a bijection, (2.4) implies (2.5).

\section{$\S 3$. Decomposition theorem on differential polynomials of theta functions}

3.1. First let us prove the algebra isomorphic theorem:

THEOREM 3.1. The replacement

$$
\Delta^{j} \varphi(z) \longrightarrow\left(\frac{\partial}{\partial z}\right)^{j} \varphi(z) \quad\left(j \in Z_{\geq 0}^{g}, \varphi \in \Theta_{0}\right)
$$

induces a $\Theta_{0}$-algebra isomorphism of $\Theta$ onto the algebra

$$
C\left[\cdots,\left(\frac{\partial}{\partial z}\right)^{j} \vartheta^{(n)}\left[\begin{array}{c}
a / n \\
0
\end{array}\right](\tau \mid z), \cdots\right]
$$

of differential polynomials of theta functions, namely

$1^{\circ} G\left(\cdots, \Delta^{j} \vartheta^{(n)}\left[\begin{array}{c}a / n \\ 0\end{array}\right](\tau \mid z), \cdots\right)=0$,

if and only if $G\left(\cdots,\left(\frac{\partial}{\partial z}\right)^{j} \vartheta^{(n)}\left[\begin{array}{c}a / n \\ 0\end{array}\right](\tau \mid z), \cdots\right)=0$,

$2^{\circ} \quad G\left(\cdots, \Delta^{j} \vartheta^{(n)}\left[\begin{array}{c}a / n \\ 0\end{array}\right](\tau \mid z), \cdots\right)=G\left(\cdots,\left(\frac{\partial}{\partial z}\right)^{j} \vartheta^{(n)}\left[\begin{array}{c}a / n \\ 0\end{array}\right](\tau \mid z), \cdots\right)$

if and only if $G\left(\cdots,\left(\frac{\partial}{\partial z}\right)^{j} \vartheta^{(n)}\left[\begin{array}{c}a / n \\ 0\end{array}\right](\tau \mid z), \cdots\right) \in \Theta_{0}$.

Proof. It is enough to assume $G\left(\cdots, \Delta^{j} \vartheta^{(n)}\left[\begin{array}{c}a / n \\ 0\end{array}\right](\tau \mid u, z), \cdots\right)$ belongs 
to $\Theta^{(m)}$ with some $m$. If $G\left(\cdots, \Delta^{j} \vartheta^{(n)}\left[\begin{array}{c}a / n \\ 0\end{array}\right](\tau \mid z), \cdots\right)=0$, then putting $u=0$, we obtain $G\left(\cdots,\left(\frac{\partial}{\partial z}\right)^{j} \vartheta^{(n)}\left[\begin{array}{c}a / n \\ 0\end{array}\right](\tau \mid z), \cdots\right)=0$. By virtue of the direct decomposition theorem we may put

$$
G\left(\cdots, \Delta^{j} \vartheta^{(n)}\left[\begin{array}{c}
a / n \\
0
\end{array}\right](\tau \mid z), \cdots\right)=\sum_{h} \Delta^{n} \phi_{h}(z)
$$

with $\phi_{h} \in \Theta_{0}^{(m)}$. If we assume $G\left(\cdots,\left(\frac{\partial}{\partial z}\right)^{j} \vartheta^{(n)}\left[\begin{array}{c}a / n \\ 0\end{array}\right](\tau \mid z), \cdots\right)=0$, then we have

$$
\begin{aligned}
\sum_{h}\left(\frac{\partial}{\partial z}\right)^{h} \phi_{h}(z) & =G\left(\cdots, \Delta^{j} \vartheta^{(n)}\left[\begin{array}{c}
a / n \\
0
\end{array}\right](\tau \mid z), \cdots\right)_{\mid u=0} \\
& =G\left(\cdots,\left(\frac{\partial}{\partial z}\right)^{j} \vartheta^{(n)}\left[\begin{array}{c}
a / n \\
0
\end{array}\right](\tau \mid z), \cdots\right)=0 .
\end{aligned}
$$

Therefore it is enough to show $\phi_{h}(z)=0$ under the condition

$$
\sum_{h}\left(\frac{\partial}{\partial z}\right)^{h} \phi_{h}(z)=0 \text { and } \quad \phi_{h}(z) \in \Theta_{0}^{(m)} \text {. }
$$

For each $b \in Z^{g}$ it follows

$$
\begin{aligned}
\phi_{h}(z+b \tau)=\exp (- & \left.\pi m \sqrt{-1}\left(b \tau^{t} b+2 z^{t} b\right)\right) \phi_{h}(z) \\
\sum_{h}\left(\frac{\partial}{\partial z}\right)^{h} \phi_{h}(z+b \tau)= & \sum_{h}\left(\frac{\partial}{\partial z}\right)^{h}\left(\exp \left(-\pi m \sqrt{-1}\left(b \tau^{t} b+2 z^{t} b\right)\right) \phi_{h}(z)\right) \\
= & \exp \left(-\pi m \sqrt{-1}\left(b \tau^{t} b+2 z^{t} b\right)\right) \sum_{h} \sum_{p}\left(\begin{array}{l}
h \\
p
\end{array}\right) \\
& \cdot(-2 \pi m \sqrt{-b})^{p}\left(\frac{\partial}{\partial z}\right)^{h-p} \phi^{h}(z) \quad\left(b \in Z^{g}\right)
\end{aligned}
$$

and thus

(*) $\quad \sum_{h} \sum_{p}\left(\begin{array}{l}h \\ p\end{array}\right)(-2 \pi m \sqrt{-1} b)^{p}\left(\frac{\partial}{\partial z}\right)^{h-p} \phi_{h}(z)=0 \quad\left(b \in Z^{g}\right)$.

Let $h_{0}$ be one of maximal $h$ in the above sum. Then, the coefficients of $b^{h_{0}}$ in the polynomial relation $(*)$ in $b$ is given by $(-2 \pi m \sqrt{-1})^{\left|h_{0}\right|} \phi_{h_{0}}(z)$, hence we may conclude $\phi_{h_{0}}(z)=0$. Proceeding this process successively we have $\phi_{h}(z)=0$, i.e. $G\left(\cdots, \Delta^{j} \vartheta^{(n)}\left[\begin{array}{c}a / n \\ 0\end{array}\right](\tau \mid z), \cdots\right)=0$. Since $G(\cdots$, $\left.\Delta^{j} \vartheta^{(n)}\left[\begin{array}{c}a / n \\ 0\end{array}\right](\tau \mid z), \cdots\right)$ belongs to $\Theta^{(m)}$, assuming 


$$
G\left(\cdots, \Delta^{j} \vartheta^{(n)}\left[\begin{array}{c}
a / n \\
0
\end{array}\right](\tau \mid z), \cdots\right)=G\left(\cdots,\left(\frac{\partial}{\partial z}\right)^{j} \vartheta^{(n)}\left[\begin{array}{c}
a / n \\
0
\end{array}\right](\tau \mid z), \cdots\right)
$$

we have

$$
\begin{aligned}
& G\left(\cdots,\left(\frac{\partial}{\partial z}\right)^{j} \vartheta^{(n)}\left[\begin{array}{c}
a / n \\
0
\end{array}\right](\tau \mid z), \cdots\right)_{\mid z \rightarrow z+\hat{b}+b \tau} \\
& \quad=G\left(\cdots, \Delta^{j} \vartheta^{(n)}\left[\begin{array}{c}
a / n \\
0
\end{array}\right](\tau \mid z), \cdots\right)_{((u, z) \rightarrow(u+b, z+\hat{b}+b \tau)} \\
& \quad=\exp \left(-\pi m \sqrt{-1}\left(b \tau^{t} b+2 z^{t} b\right)\right) G\left(\cdots, \Delta^{j} \vartheta^{(n)}\left[\begin{array}{c}
a / n \\
0
\end{array}\right](\tau \mid z), \cdots\right) \\
& \quad=\exp \left(-\pi m \sqrt{-1}\left(b \tau^{t} b+2 z^{t} b\right)\right) G\left(\cdots,\left(\frac{\partial}{\partial z}\right)^{j} \vartheta^{(n)}\left[\begin{array}{c}
a / n \\
0
\end{array}\right](\tau \mid z), \cdots\right)
\end{aligned}
$$

i.e.

$$
G\left(\cdots,\left(\frac{\partial}{\partial z}\right)^{j} \vartheta^{(n)}\left[\begin{array}{c}
a / n \\
0
\end{array}\right](\tau \mid z), \cdots\right) \in \Theta_{0}^{(m)} .
$$

Conversely, if

$$
G\left(\cdots,\left(\frac{\partial}{\partial z}\right)^{j} \vartheta^{(n)}\left[\begin{array}{c}
a / n \\
0
\end{array}\right](\tau \mid z), \cdots\right) \in \Theta_{0}^{(m)}
$$

then applying $1^{\circ}$ for

$$
\begin{aligned}
& F\left(\cdots, \Delta^{j} \vartheta^{(n)}\left[\begin{array}{c}
a / n \\
0
\end{array}\right](\tau \mid z), \cdots\right) \\
& \quad=G\left(\cdots, \Delta^{j} \vartheta^{(n)}\left[\begin{array}{c}
a / n \\
0
\end{array}\right](\tau \mid z), \cdots\right)-G\left(\cdots,\left(\frac{\partial}{\partial z}\right)^{j} \vartheta^{(n)}\left[\begin{array}{c}
a / n \\
0
\end{array}\right](\tau \mid z), \cdots\right)
\end{aligned}
$$

we obtain

$$
F\left(\cdots, \Delta^{j} \vartheta^{(n)}\left[\begin{array}{c}
a / n \\
0
\end{array}\right](\tau \mid z), \cdots\right)=0
$$

i.e.

$$
G\left(\cdots, \Delta^{j} \vartheta^{(n)}\left[\begin{array}{c}
a / n \\
0
\end{array}\right](\tau \mid z), \cdots\right)=G\left(\cdots,\left(\begin{array}{c}
\partial \\
\partial z
\end{array}\right)^{j} \vartheta^{(n)}\left[\begin{array}{c}
a / n \\
n
\end{array}\right](\tau \mid z), \cdots\right) .
$$

Combining Theorem 2.1 and Theorem 3.1 we obtain the decomposition theorem.

TheOREM 3.2. The algebra $C\left[\cdots,\left(\frac{\partial}{\partial z}\right)^{j} \vartheta^{(n)}\left[\begin{array}{c}a / n \\ 0\end{array}\right](\tau \mid z), \cdots\right]$ of differential polynomials of theta functions has a canonical linear basis 


$$
\left\{\left(\frac{\partial}{\partial z}\right)^{j} \vartheta^{(n)}\left[\begin{array}{c}
a / n \\
0
\end{array}\right](\tau \mid z) \mid j \in Z_{\geq 0}^{g}, a \in Z^{g} / n Z^{g}, n \geq 1\right\},
$$

namely differential polynomials of theta functions are uniquely expressed as linear combinations of (3.1) with constant coefficients depending on $\tau$.

3.2. In order to express the decomposition of differential polynomials of theta functions explicitly, we introduce differential polynomials in $Y_{1}, \cdots, Y_{r}$

$$
\begin{aligned}
& F_{j^{(1)}, \ldots, j(r) ; h}^{\left(n_{1}, \ldots, n_{r}\right)}\left(Y_{1}, \cdots, Y_{r} \mid z\right) \\
& =\frac{1}{h !\left(n_{1}+\cdots+n_{r}\right)^{|h|}} \sum_{p} \frac{(-1)^{|p|}}{p !}\left(\frac{1}{n_{1}+\cdots+n_{r}} \frac{\partial}{\partial z}\right)^{p} \\
& \text { - }\left\{\sum_{\substack{k^{(1)}+\ldots \tilde{k}^{+(\alpha)} \leq j^{(r)}=p+h \\
j^{(\alpha)}}}\left(\frac{p+h}{k^{(1)}, \cdots, k^{(r)}}\right) \frac{1}{\left(j^{(1)}-k^{(1)}\right) !} \cdots \frac{1}{\left(j^{(1)}-k^{(r)}\right) !}\right. \\
& \left.\cdot\left(\frac{1}{n_{1}} \frac{\partial}{\partial z}\right)^{j^{(1)-k^{(1)}}} Y_{1} \cdots\left(\frac{1}{n_{r}} \frac{\partial}{\partial z}\right)^{j^{(r)}-k^{(r)}} Y_{r}\right\} \\
& \left(j^{(1)}, \cdots, j^{(r)}, h \in Z_{\geq 0}^{g}, n_{1}, \cdots, n_{r} \geq 1\right) .
\end{aligned}
$$

THeOREM 3.3. For theta functions $\varphi_{\alpha}(z) \in \Theta_{0}^{\left(n_{\alpha}\right)}(1 \leq \alpha \leq r) F_{j(1), \ldots, n_{j}(r) ; h}^{\left(n_{1}, \ldots, n_{r}\right.}$ $\times\left(\varphi_{1}, \cdots, \varphi_{r} \mid z\right),\left(j^{(1)}, \cdots, j^{(r)}, h \in Z_{Z_{0}}^{g}\right)$ are theta functions of level $n_{1}+\cdots$ $+n_{r}$ such that

$$
\begin{aligned}
\frac{1}{j^{(1)} ! \cdots j^{(r) !}}\left(\frac{1}{n_{1}} \frac{\partial}{\partial z}\right)^{j(1)} \varphi_{1}(z) \cdots\left(\frac{1}{n_{r}} \frac{\partial}{\partial z}\right)^{j(r)} \varphi_{r}(z) \\
=\sum_{h \leq j^{(1)}+\cdots+j^{(r)}}\left(\frac{\partial}{\partial z}\right)^{n} F_{j(1), \cdots, j(r) ; h}^{\left(n_{1}, \cdots, n_{r}\right)}\left(\varphi_{1}, \cdots, \varphi_{r} \mid z\right) \\
=\sum_{c} \lambda_{\left(j^{(1)}, \ldots, j^{(r)} ; n\right), c /\left(n_{1}+\cdots+n_{r}\right)}\left(\varphi_{1}, \cdots, \varphi_{r}\right)\left(\frac{\partial}{\partial z}\right)^{h} \vartheta^{\left(n_{1}+\cdots+n_{r}\right)} \\
\quad \cdot\left[\begin{array}{c}
c /\left(n_{1}+\cdots+n_{r}\right) \\
0
\end{array}\right](\tau \mid z),
\end{aligned}
$$

where

$$
\begin{aligned}
& \lambda_{\left(j^{(1)}, \cdots, j(r) ; h\right), c /\left(n_{1}+\cdots+n_{r}\right)}\left(\varphi_{1}, \cdots, \varphi_{r}\right) \\
&=\frac{1}{\left(n_{1}+\cdots+n_{r}\right)^{g}} \sum_{\bar{c} \in \boldsymbol{Z}^{g} /\left(n_{1}+\cdots+n_{r}\right) \boldsymbol{Z} \boldsymbol{g}} \exp \frac{2 \pi \sqrt{-1} \hat{c}^{t} c}{n_{1}+\cdots+n_{r}} \\
& \quad \cdot \vartheta^{\left(n_{1}+\cdots+n_{r}\right)}\left[\begin{array}{c}
c /\left(n_{1}+\cdots+n_{r}\right) \\
0
\end{array}\right](\tau \mid 0)^{-1} F_{j(1), \cdots(r) ; h}^{\left(n_{1}, \cdots, n_{r}\right)} \\
& \cdot\left(\varphi_{1}, \cdots, \varphi_{r} \mid \begin{array}{c}
\hat{c} \\
n_{1}+\cdots+n_{r}
\end{array}\right) .
\end{aligned}
$$


Proof. Putting

$$
\begin{aligned}
& \frac{1}{j^{(1)} ! \cdots j^{(r) !}}\left(\frac{1}{n_{1}} \Delta\right)^{j^{(1)}} \varphi_{1}(z) \cdots\left(\frac{1}{n_{r}} \Delta\right)^{j^{(r)}} \varphi_{r}(z) \\
& =\sum \Delta^{h} \psi_{h}(z)
\end{aligned}
$$

with $\psi_{h}(z) \in \Theta_{0}^{\left(n_{1} \cdots+n_{r}\right)}$, by virtue of Corollary of Theorem 2.1 (1.6) and (1.7) we have

$$
\begin{aligned}
& \psi_{h}(z)=\frac{1}{h !\left(n_{1}+\cdots+n_{r}\right)^{|h|}} \sum_{p} \frac{(-1)^{|p|}}{p !}\left(\frac{1}{n_{1}+\cdots+n_{r}} \Delta\right)^{p} \mathscr{D}^{p+h} \\
& \cdot \frac{1}{j^{(1)} ! \cdots j^{(r) !}}\left(\frac{1}{n_{1}} \Delta\right)^{j^{(1)}} \varphi_{1}(z) \cdots\left(\frac{1}{n_{r}} \Delta\right)^{j^{(r)}} \varphi_{r}(z) \\
& =\frac{1}{h !\left(n_{1}+\cdots+n_{r}\right)^{|h|}} \sum_{p} \frac{(-1)^{|p|}}{p !}\left(\frac{1}{n_{1}+\cdots+n_{r}} \Delta\right)^{p}
\end{aligned}
$$

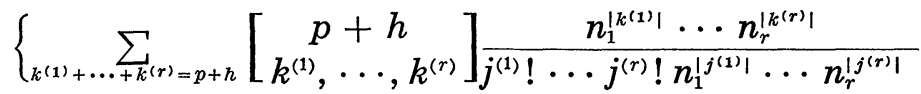

$$
\begin{aligned}
& \left.\cdot n^{-\left|k^{(1)}\right|} \mathscr{D}^{k^{(1)}} \Delta^{j(1)} \varphi_{1}(z) \cdots n^{-\left|k^{(r)}\right|} \mathscr{D}^{k(r)} \Delta^{j(r)} \varphi_{r}(z)\right\} \\
& =\frac{1}{h !\left(n_{1}+\cdots+n_{r}\right)^{|h|}} \sum_{p} \frac{(-1)^{|p|}}{p !}\left(\frac{1}{n_{1}+\cdots+n_{r}} \Delta\right)^{p}
\end{aligned}
$$

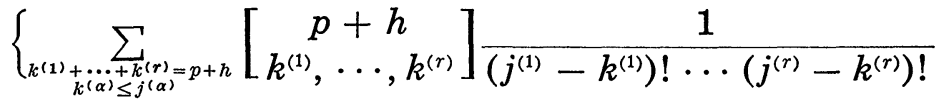

$$
\begin{aligned}
& \left.\cdot\left(\frac{1}{n_{1}} \Delta\right)_{\varphi_{1}(z)}^{j^{(1)}-k^{(1)}} \cdots\left(\frac{1}{n_{r}} \Delta\right)_{\varphi_{r}(z)}^{j^{(r)}-k^{(r)}}\right\} \\
& =F_{j(1), \ldots, j(r) ; n}^{\left(n_{1}, \ldots, n_{r}\right)}\left(\varphi_{1}, \cdots, \varphi_{r} \mid z\right) \text {. }
\end{aligned}
$$

Hence, replacing $\Delta_{i}$ by $\partial / \partial z_{i}(1 \leq i \leq g)$, we prove the first assertion of Theorem 3.3. Putting

$$
\begin{aligned}
& F_{j(1, \cdots, j(r) ; h}^{\left(n_{1}, \ldots n_{r}\right)}\left(\varphi_{1}, \cdots, \varphi_{r} \mid z\right) \\
& =\sum_{c \in Z^{g} /\left(n_{1}+\cdots+n_{r}\right) Z^{g}} \lambda_{h, c} \vartheta^{\left(n_{1}+\cdots+n_{r}\right)}\left[\begin{array}{c}
c /\left(n_{1}+\cdots+n_{r}\right) \\
0
\end{array}\right](\tau \mid z),
\end{aligned}
$$

we have

$$
\begin{aligned}
& F_{j^{(1)}, \cdots, \cdots, j(r) ; h}^{\left(n_{1}, \cdots, n_{r}\right)}\left(\varphi_{1}, \cdots, \varphi_{r} \mid \frac{\hat{c}}{n_{1}+\cdots+n_{r}}\right) \\
& =\sum_{c} \lambda_{h, c} \vartheta^{\left(n_{1}+\cdots+n_{r}\right)}\left[\begin{array}{c}
c /\left(n_{1}+\cdots+n_{r}\right) \\
0
\end{array}\right]\left(\tau \mid \frac{\hat{c}}{n_{1}+\cdots+n_{r}}\right) \\
& =\sum_{c} \lambda_{h, c} \exp \left(\frac{2 \pi \sqrt{-1} \hat{c}^{t} c}{n_{1}+\cdots+n_{r}}\right) \vartheta^{\left(n_{1}+\cdots+n_{r}\right)}\left[c /\left(n_{1}+\cdots+n_{r}\right)\right](\tau \mid 0) \\
& 0 \\
& \left(c \in Z^{g} /\left(n_{1}+\cdots+n_{r}\right) Z^{g}\right) .
\end{aligned}
$$


Hence, by virtue of the orthogonal relation for characters

$$
\sum_{c} \exp \left(\frac{2 \pi \sqrt{-1} \hat{c}^{t} c}{n_{1}+\cdots+n_{r}}\right)=\left\{\begin{array}{ccc}
\left(n_{1}+\cdots+n_{r}\right)^{g} & \hat{c} \equiv 0 & \bmod \left(n_{1}+\cdots+n_{r}\right) \\
0 & \hat{c} \neq 00 & \bmod \left(n_{1}+\cdots+n_{r}\right),
\end{array}\right.
$$

it follows

$$
\begin{aligned}
\lambda_{h, c}= & \frac{1}{\left(n_{1}+\cdots+n_{r}\right)^{g}} \sum_{\hat{c}} \exp \left(\frac{-2 \pi \sqrt{-1} \hat{c}^{t} c}{n_{1}+\cdots+n_{r}}\right) \vartheta^{\left(n_{1}+\cdots+n_{r}\right)} \\
& \cdot\left[\begin{array}{c}
c /\left(n_{1}+\cdots+n_{r}\right) \\
0
\end{array}\right](\tau \mid 0)^{-1} F_{j(1), \cdots, j(r) ; n}^{\left(n_{1}, \cdots, n_{r}\right)}\left(\varphi_{1}, \cdots, \varphi_{r} \mid \frac{\hat{c}}{n_{1}+\cdots+n_{r}}\right) .
\end{aligned}
$$

Specializing

$$
\left(\varphi_{1}(z), \varphi_{2}(z)\right) \quad \text { to } \quad\left(\vartheta^{\left(n_{1}\right)}\left[\begin{array}{c}
a_{1} / n_{1} \\
0
\end{array}\right](\tau \mid z), \vartheta^{\left(n_{r}\right)}\left[\begin{array}{c}
a_{r} / n_{r} \\
0
\end{array}\right](\tau \mid z)\right)
$$

we obtain the explicit expression of structure constants of

$$
C\left[\cdots,\left(\frac{\partial}{\partial z}\right)^{j} \vartheta^{(n)}\left[\begin{array}{c}
a / n \\
0
\end{array}\right](\tau \mid z), \cdots\right]
$$

with respect to the basis

$$
\left\{\left(\frac{\partial}{\partial z}\right)^{j} \vartheta^{(n)}\left[\begin{array}{c}
a / n \\
0
\end{array}\right](\tau \mid z)\right\}
$$

THEOREM 3.4. The structure constants of

$$
C\left[\cdots,\left(\frac{\partial}{\partial z}\right)^{j} \vartheta^{(n)}\left[\begin{array}{c}
a / n \\
0
\end{array}\right](\tau \mid z), \cdots\right]
$$

are given by

$$
\begin{aligned}
& \left(\frac{\partial}{\partial z}\right)^{j(1)} \vartheta^{\left(n_{1}\right)}\left[\begin{array}{c}
a_{1} / n_{1} \\
0
\end{array}\right](\tau \mid z)\left(\frac{\partial}{\partial z}\right)^{j(2)} \vartheta^{\left(n_{2}\right)}\left[\begin{array}{c}
a_{2} / n_{2} \\
0
\end{array}\right](\tau \mid z) \\
& =\sum_{h} \sum_{c} \gamma_{\left(j(1), a_{1} / n_{2}, n_{1}\right),\left(j(2), a_{2} / n_{2}, n_{2}\right)}^{\left(h, c /\left(n_{1}+n_{2}\right), n_{1}+n_{2}\right)}(\tau)\left(\frac{\partial}{\partial z}\right)^{h} \vartheta^{\left(n_{1}+n_{2}\right)}\left[\begin{array}{c}
c /\left(n_{1}+n_{2}\right) \\
0
\end{array}\right](\tau \mid z), \\
& \gamma_{\left(j(1), a_{1} n_{1}, n_{1}\right)\left(j(2), a_{2} / n_{2}, n_{2}\right)}^{(h, c)(\tau)} \\
& =\frac{j^{(1)} ! j^{(2)} ! n_{1}^{|j(1)|} n_{2}^{\left|j^{(2)}\right|}}{h !\left(n_{1}+n_{2}\right)^{g+|h|}} \sum_{\hat{c} \in Z^{g} /\left(n_{1}+n_{2}\right) Z_{n}^{g}} \exp \left(\frac{-2 \pi \sqrt{-1} \hat{c}^{t} c}{n_{1}+n_{2}}\right) \vartheta^{\left(n_{1}+n_{2}\right)} \\
& \cdot\left[\begin{array}{c}
c /\left(n_{1}+n_{2}\right) \\
0
\end{array}\right](\tau \mid 0)^{-1}\left[\sum_{p} \frac{(-1)^{|p|}}{p !}\left(\frac{1}{n_{1}+n_{2}} \frac{\partial}{\partial z}\right)^{p} \sum_{\substack{k(1)+k^{(2)}=p+h \\
k(1) \leq j(1), k^{(2)} \leq j^{(2)}}}\right. \\
& \cdot\left[\begin{array}{c}
p+h \\
k^{(1)}, k^{(2)}
\end{array}\right] \frac{1}{\left(j^{(1)}-k^{(1)}\right) !\left(j^{(2)}-k^{(2)}\right) !}\left(\frac{1}{n_{1}} \frac{\partial}{\partial z}\right)^{j^{(1)}-k^{(1)}} \vartheta^{\left(n_{1}\right)}\left[\begin{array}{c}
a_{1} / n_{1} \\
0
\end{array}\right]
\end{aligned}
$$




$$
\left.\cdot(\tau \mid z)\left(\frac{1}{n_{2}} \frac{\partial}{\partial z}\right)^{j^{(2)-k(2)}} \vartheta^{\left(n_{2}\right)}\left[\begin{array}{c}
a_{2} / n_{2} \\
0
\end{array}\right](\tau \mid z)\right]_{z=\hat{c}\left(n_{1}+n_{2}\right)} .
$$

For theta functions $\varphi_{a}(z)(1 \leq \alpha \leq z)$, if a differential polynomial $G\left(\cdots,(\partial / \partial z)^{j} \varphi_{\alpha}(z), \cdots\right)$ is a theta function, then by virtue of Theorems 2.1 and $3.1 G\left(\cdots,(\partial / \partial z)^{j} \varphi_{\alpha}(z), \cdots\right)$ is itself the $\Theta_{0}$-component of the decomposition. Hence, Theorem 3.4 implies the following characterization of diffreential polynomials of $\varphi_{\alpha}(z)(1 \leq \alpha \leq r)$ which are also theta functions.

THEOREM 3.5. For theta functions $\varphi_{\alpha}(z) \in \Theta_{0}^{\left(n_{\alpha}\right)}$ the space

$$
C\left[\cdots,\left(\frac{\partial}{\partial z}\right)^{j} \varphi_{\alpha}(z), \cdots\right] \cap \Theta_{0}^{(m)}
$$

is linearly spanned by

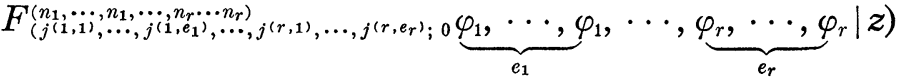

$$
\begin{aligned}
& \left(\sum_{\alpha} e_{\alpha} n_{\alpha}=m ; j^{(1,1)}, \cdots, j^{\left(1, e_{1}\right)}, \cdots, j^{(r, 1)}, \cdots,{ }^{\left(r, e_{r}\right)} \in Z_{\geq 0}^{g}\right) .
\end{aligned}
$$

\section{REFERENCES}

[1] R. Hirota, A direct method of finding exact solution of nonlinear evolution equations, Lecture Notes in Mathematics, No. 515, 40-68 (1976).

[2] H. Morikawa, Some analytic and geometric applications of the invariant theoretic method, Nagoya Math. J., 80 (1980), 1-47.

[ 3 ] — On Poisson brackets of semi-invariants, Manifolds and Lie groups, 267-281, Progress in Math. Birkhänser (1981).

Department of Mathematics

Faculty of Science

Nagoya University

Chikusa-ku 464

Japan 